UCRL-CONF-204710

June 15, 2004

17h ASCE Engineering Mechanics Conference

University of Delaware, Newark, DE, June 14-16, 2004

\title{
COMPARISON OF UP-SCALING METHODS IN POROELASTICITY AND ITS GENERALIZATIONS
}

\author{
James G. Berryman \\ University of California \\ Lawrence Livermore National Laboratory \\ Livermore, $C A$
}


This document was prepared as an account of work sponsored by an agency of the United States Government. Neither the United States Government nor the University of California nor any of their employees, makes any warranty, express or implied, or assumes any legal liability or responsibility for the accuracy, completeness, or usefulness of any information, apparatus, product, or process disclosed, or represents that its use would not infringe privately owned rights. Reference herein to any specific commercial product, process, or service by trade name, trademark, manufacturer, or otherwise, does not necessarily constitute or imply its endorsement, recommendation, or favoring by the United States Government or the University of California. The views and opinions of authors expressed herein do not necessarily state or reflect those of the United States Government or the University of California, and shall not be used for advertising or product endorsement purposes. 


\section{OUTLINE}

- Biot's Poroelasticity Theory Is Correct!

- Laboratory data

○ Finite element approach to modeling

- Four Methods for Up-Scaling

○ Effective medium theories

$\circ$ Mixture theory

- Homogenization theory

○ Volume averaging methods

- Conclusions 


\section{Biot's (1962) Strain Energy Functional}

$$
2 E=H e^{2}-2 C e \zeta+M \zeta^{2}-4 \mu I_{2}
$$

where $H, C, M$, and $\mu$ are poroelastic constants,

$e=\nabla \cdot \vec{u}=$ frame dilatation,

$\zeta=-\nabla \cdot \vec{w}=$ increment of fluid content,

$\phi=$ porosity,

$\vec{u}=$ solid frame displacement,

$\vec{u}_{f}=$ pore fluid displacement,

$\vec{w}=\phi\left(\vec{u}_{f}-\vec{u}\right)=$ relative displacement, and

$I_{2}=e_{x} e_{y}+e_{y} e_{z}+e_{z} e_{x}-\frac{1}{4}\left(\gamma_{x}^{2}+\ldots\right)=$ a strain invariant. 


\section{Biot's Equations of Dynamic Poroelasticity}

$$
\begin{gathered}
\omega^{2} \rho \vec{u}+(H-\mu) \nabla e+\mu \nabla^{2} \vec{u}=-\omega^{2} \rho_{f} \vec{w}+C \nabla \zeta, \\
\omega^{2} q(\omega) \vec{w}-M \nabla \zeta=-\omega^{2} \rho_{f} \vec{u}-C \nabla e,
\end{gathered}
$$

where

$\omega=2 \pi f=$ angular frequency,

$\rho=\phi \rho_{f}+(1-\phi) \rho_{m}=$ the average density,

$q(\omega)=\rho_{f}[\tau / \phi+i F(\xi) \eta / \kappa \omega]$, and

$p_{f}=-M \nabla \cdot \vec{w}-C \nabla \cdot \vec{u}=$ fluid pressure. 


\section{Some Relations Among Poroelastic Constants}

$$
\begin{gathered}
H=K_{u}+\frac{4}{3} \mu, \\
C=B K_{u}, \\
M=B K_{u} / \alpha=C / \alpha,
\end{gathered}
$$

where

$\alpha=1-K / K_{m}=$ the effective stress coefficient, and

$K_{u}$ is the undrained or Gassmann bulk modulus of the system. 


\section{Dispersion Relations}

- For shear wave:

$$
k_{s}^{2}=\omega^{2}\left(\rho-\rho_{f}^{2} / q\right) / \mu
$$

- For fast and slow compressional waves:

$$
\begin{gathered}
k_{ \pm}^{2}=\frac{1}{2}\left[b+f \mp\left[(b-f)^{2}+4 c d\right]^{1 / 2}\right] \\
b=\omega^{2}\left(\rho M-\rho_{f} C\right) / \Delta, \quad c=\omega^{2}\left(\rho_{f} M-q C\right) / \Delta \\
d=\omega^{2}\left(\rho_{f} H-\rho C\right) / \Delta, \quad f=\omega^{2}\left(q H-\rho_{f} C\right) / \Delta
\end{gathered}
$$

where

$$
\Delta=H M-C^{2} .
$$




\section{SOME UP-SCALING RESULTS}

via effective medium theory or homogenization methods

- Electrical Conductivity (scale invariant)

$$
J=\sigma E \rightarrow\langle J\rangle=\sigma^{*}\langle E\rangle
$$

- Navier-Stokes equation $\rightarrow$ Darcy's equation

$$
\text { definitely not scale invariant! }
$$

- Linear elasticity + Navier-Stokes equations $\rightarrow$

Biot's equations of poroelasticity

- Heterogeneous Biot $\rightarrow$ ????

Possibly to the double-porosity model in a variety of circumstances (last year's talk!). 


\section{First Method: Effective Medium Theory}

Effective medium theory is designed to produce

estimates of coefficients in the equations of motion.

Various good alternatives are available:

o Average T-matrix (Mori-Tanaka, Kuster-Toksöz)

- Self-consistent (SC or CPA)

- Differential effective medium (DEM)

○ Also, rigorous bounding methods are known. 


\section{Second Method: Mixture Theory}

Mixture theory is designed to keep careful track of the energy in the system. So this

approach includes:

- Hamiltonian and Lagrangian methods

- Biot's original method

○ Drumheller and Bedford's method

This method is especially powerful for nonlinear problems, but also provides a good method to derive Biot's linear equations. 


\section{Third Method: Homogenization Theory}

Homogenization theory is probably the newest

of the methods, being first developed in the

1970s. Other methods can be traced back to earlier

periods of history. Periodic boundary conditions

are normally used to implement the method.

Development is designed to determine rigorously

the form of the equations in some fixed

frequency regime. So it may not determine how

the equations change as frequency is varied widely. 


\section{Fourth Method: Volume Averaging}

Volume averaging was apparently first developed

in the 1960s for application to Darcy flow.

There are similarities to homogenization theory,

but does not require periodic boundary conditions.

Uses rigorous identities concerning volume integration in $3 \mathrm{D}$ to smooth the equations of interest.

Not restricted to a fixed frequency domain,

but requires supplementary information to obtain estimates of the coefficients. 


\section{Other Methods: Were Any Left Out?}

- There are other methods I have not talked about today, including:

- Double-porosity up-scaling (my talk last year)

○ Numerical methods

- Hybrid methods - using two or more methods simultaneously: for example, mixture theory supplemented with effective medium theory is one very powerful combination. 


\section{CONCLUSIONS}

I take a very democractic viewpoint concerning

all these methods. I have never seen an up-scaling

method I did not like. (Well, almost never!)

All these up-scaling methods have some advantages

and some disadvantages.

I have stressed the advantages today. 


\section{ACKNOWLEDGMENT}

This work was performed under the auspices of the U.S.

Department of Energy by the University of California,

Lawrence Livermore National Laboratory under contract

No. W-7405-ENG-48 and supported specifically by the

Geosciences Research Program of the DOE Office of Energy

Research within the Office of Basic Energy Sciences.

All support of the work is gratefully acknowledged. 